\title{
Synthesis of a mannose heptasaccharide of the pathogenic yeast, Candida glabrata IFO 0622 strain
}

\author{
Youlin Zeng, Jianjun Zhang, Fanzuo Kong* \\ Research Center for Eco-Environmental Sciences, Academia Sinica, Chinese Academy of Sciences, PO Box 2871, Beijing 100085, PR China
}

Received 12 April 2002; accepted 9 May 2002

\begin{abstract}
An effective synthesis of the mannose heptasaccharide existing in the pathogenic yeast, Candida glabrata IFO 0622 strain was achieved via TMSOTf-promoted condensation of a tetrasaccharide donor $\mathbf{1 3}$ with a trisaccharide acceptor 16, followed by deprotection. The tetrasaccharide 13 was constructed by coupling of 2,3,4,6-tetra- $O$-benzoyl- $\alpha$-D-mannopyranosyl-(1 $\rightarrow 3)-2,4,6-$ tri- $O$-acetyl- $\alpha$-D-mannopyranosyl trichloroacetimidate (7) with allyl 3,4,6-tri- $O$-benzoyl- $\alpha$-D-mannopyranosyl-(1 $\rightarrow 2)-3,4,6$-tri- $O$ benzoyl- $\alpha$-D-mannopyranoside (10), followed by deallylation and trichloroacetimadation. The trisaccharide $\mathbf{1 6}$ was obtained by coupling of 6- $O$-acetyl-2,3,4-tri- $O$-benzoyl- $\alpha$-D-mannopyranosyl trichloroacetimidate with 10, and subsequent 6-O-deacetylation. The disaccharide 7 was prepared through coupling of perbenzoylated mannosyl trichloroacetimidate with 4,6-O-benzylidene-1,2$O$-ethylidene- $\beta$-D-mannopyranose, then simultaneous debenzylidenation and deethylidenation, and subsequent acetylation, selective 1-O-deacetylation, and trichloroacetimidation. The disaccharide $\mathbf{1 0}$ was obtained by self-condensation of 3,4,6-tri- $O$-benzoyl-1,2- $O$-allyloxyethylidene- $\beta$-D-mannopyranose, followed by selective 2-O-deacetylation. C 2002 Elsevier Science Ltd. All rights reserved.
\end{abstract}

Keywords: Mannose oligosaccharides; Trichloroacetimidates; Regio- and stereoselective synthesis

\section{Introduction}

Candida species are opportunistic pathogens of humans that frequently cause severe systemic infections in patients with AIDS, ${ }^{1}$ cancer, ${ }^{2}$ and burns ${ }^{3}$ as well as in those under immunosuppressive or radiation therapy. ${ }^{4}$ Candida glabrata as a medically important fungus has been steadily attracting attention from microbiologists interested in infectious disease research. ${ }^{5}$ A structural analysis of the cell wall mannan isolated from $C$. glabrata IFO 0622 strain was carried out by Suzuki's group. ${ }^{6}$ Three novel oligosaccharides, i.e., a tetraose, a hexaose, and a heptaose, were obtained from mild acetolysis of acid- and alkali-stable mannan moiety. The tetraose and hexaose were known $\alpha-(1 \rightarrow 2)$ - and $\alpha-(1 \rightarrow 3)$-linked structures, while the heptaose was identified as $\alpha$-D-Man $p-(1 \rightarrow 3)-\alpha-\mathrm{D}-\operatorname{Man} p-(1 \rightarrow 2)-\alpha-\mathrm{D}-$ $\operatorname{Man} p-(1 \rightarrow 2)-\alpha-\mathrm{D}-\operatorname{Man} p-(1 \rightarrow 6)-\alpha-\mathrm{D}-\operatorname{Man} p-(1 \rightarrow 2)-\alpha-$

\footnotetext{
* Corresponding author. Tel.: + 86-10-62936613; fax: + 86-10-62923563

E-mail address: fzkong@mail.rcees.ac.cn (F. Kong).
}

D-Man $p-(1 \rightarrow 2)$-D-Man. To the best of our knowledge, there have been no reports dealing with the synthesis of the heptasaccharide. For an investigation of structurefunction relationships of mannan, we present herein a facile and convergent synthesis of the mannose heptasaccharide.

\section{Results and discussion}

Retrosynthetic analysis indicated that the mannose heptamer can be obtained with a $(1 \rightarrow 6)$ linkage by condensation of two moieties, i.e., a mannose tetramer donor and a mannose trimer acceptor. The tetraasaccharide then can be constructed from a $(1 \rightarrow 3)$ linked disaccharide donor and a $(1 \rightarrow 2)$-linked disaccharide acceptor, while the trisaccharide can be built from the same $(1 \rightarrow 2)$-linked disaccharide acceptor and a mannose donor.

Our synthetic route is shown in Scheme 1. Coupling of perbenzoylated mannosyl trichloroacetimidate ${ }^{7} \mathbf{1}$ with 4,6- $O$-benzylidene-1,2- $O$-ethylidene- $\beta$-D-mannopy- 


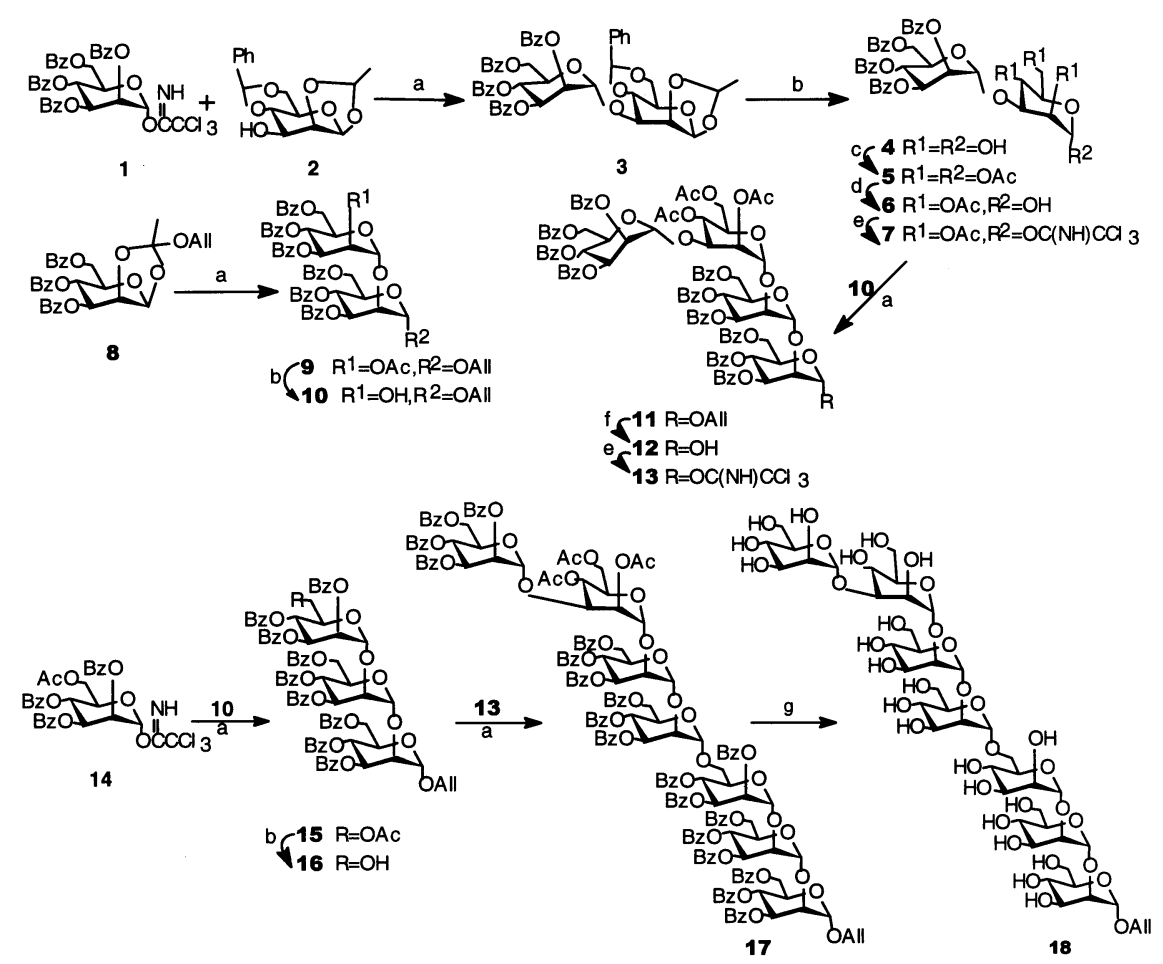

Scheme 1. Reagents and conditions: a. TMSOTf, $\mathrm{CH}_{2} \mathrm{Cl}_{2}, \mathrm{~N}_{2},-15^{\circ} \mathrm{C}$ to rt, 4 h; b. $\mathrm{CH}_{2} \mathrm{Cl}_{2}, \mathrm{CH}_{3} \mathrm{OH}-\mathrm{CH} \mathrm{COCl}_{3}$, rt; c. Ac $\mathrm{O}_{2}$ py (dry), rt, 6 h; d. $\mathrm{CH}_{2} \mathrm{Cl}_{2}, \mathrm{NH}_{3}-\mathrm{MeOH}$, rt; e. $\mathrm{CCl}_{3} \mathrm{CN}, \mathrm{DBU}, \mathrm{CH}_{2} \mathrm{Cl}_{2}, \mathrm{rt}, 8$ h; f. $\mathrm{PdCl}_{2}, \mathrm{CH}_{2} \mathrm{Cl}_{2}, \mathrm{rt}, 2$ h; g. NH${ }_{3}-\mathrm{MeOH}_{\text {, rt }}$

ranose (2) gave 2,3,4,6-tetra- $O$-benzoyl- $\alpha$-D-mannopyranosyl-( $1 \rightarrow 3)-4,6$ - $O$-benzylidene-1,2- $O$-ethylidene- $\beta$ D-mannopyranose (3) in high yield (83\%). Removal of the benzylidene and ethylidene groups was readily achieved simultaneously with $0.3: 50: 10$ acetyl chloridemethanol-dichloromethane within $1 \mathrm{~h}$, giving the disaccharide 4 as a white solid in high yield $(90 \%)$ after purification. This reaction was smooth and easily controlled. Acetylation of $\mathbf{4}$ with acetic anhydride in pyridine, followed by selective 1-O-deacetylation with $\mathrm{M}$ solution of ammonia in 1:1 methanol-dichloromethane, and then trichloroacetimidation with trichloroacetonitrile in the presence of DBU furnished $2,3,4,6$ - tetra- $O$-benzoyl- $\alpha$-D-mannopyranosyl- $(1 \rightarrow 3)$ $2,4,6$-tri- $O$-acetyl- $\alpha$-D-mannopyranosyl trichloroacetimidate (7) in $61 \%$ yield (for three steps). The disaccharide 10 was prepared by TMSOTf-promoted self condensation of 3,4,6-tri- $O$-benzoyl-1,2- $O$-allyloxyethylidene- $\beta$ D-mannopyranose, ${ }^{8}$ followed by selective 2-Odeacetylation with $0.5: 100: 50$ acetyl chloridemethanol-dichloromethane. ${ }^{8,9}$ Condensation of the disaccharide donor 7 with the disaccharide acceptor $\mathbf{1 0}$ gave the tetrasaccharide $\mathbf{1 1}$ in $61 \%$ yield. Deallylation ${ }^{10}$ of 11 with $\mathrm{PdCl}_{2}$ in dichloromethane furnished the tetrasaccharide hemiacetal $\mathbf{1 2}$, and subsequent trichloroacetimidation produced the tetrasaccharide donor 13. Coupling of 6-O-acetyl-2,3,4-tri- $O$-benzoyl$\alpha$-D-mannopyranosyl trichloroacetimidate ${ }^{11}$ (14) with the disaccharide acceptor $\mathbf{1 0}$, followed by selective 6-O- deacetylation produced the trisaccharide acceptor $\mathbf{1 6}$. Finally, condensation of the tetrasaccharide donor $\mathbf{1 3}$ with 16 gave the heptasaccharide 17, and subsequent deacylation in ammonia-saturated methanol yielded the target mannose heptasaccharide $\mathbf{1 8}$, whose bioassay is in progress.

Compared to the previously reported syntheses ${ }^{10,12}$ of complex mannans containing $(1 \rightarrow 2),(1 \rightarrow 3)$, and $(1 \rightarrow$ 6) linkages, the method presented herein is simpler and convergent, owing to the sole use of acyl protection groups. It should be possible to carry out large-scale synthesis employing the method as described.

\section{Experimental}

General methods.-Optical rotations were determined at $25^{\circ} \mathrm{C}$ with a Perkin-Elmer model 241-Mc automatic polarimeter. ${ }^{1} \mathrm{H},{ }^{13} \mathrm{C}$ NMR and ${ }^{1} \mathrm{H}-{ }^{13} \mathrm{C}$ COSY spectra were recorded with Bruker ARX 400 spectrometers $\left(400 \mathrm{MHz}\right.$ for ${ }^{1} \mathrm{H}, 100 \mathrm{MHz}$ for ${ }^{13} \mathrm{C}$ ) at $25^{\circ} \mathrm{C}$ for solutions in $\mathrm{CDCl}_{3}$ or $\mathrm{D}_{2} \mathrm{O}$ as indicated. Mass spectra were measured with MALDITOF-MS spectrometer or recorded with a VG PLATFORM mass spectrometer using the ESI mode. Thin-layer chromatography (TLC) was performed on Silica Gel $\mathrm{HF}_{254}$ with detection by charring with $30 \%(\mathrm{v} / \mathrm{v}) \mathrm{H}_{2} \mathrm{SO}_{4}$ in $\mathrm{MeOH}$ or in some cases by a UV lamp. Column chromatography was conducted by elution of a column 
$(16 \times 240 \mathrm{~mm}, 18 \times 300 \mathrm{~mm}, 35 \times 400 \mathrm{~mm})$ of silica gel (100-200 mesh) with EtOAc-petroleum ether (60$90{ }^{\circ} \mathrm{C}$ ) as the eluent. Solutions were concentrated at $<60{ }^{\circ} \mathrm{C}$ under reduced pressure. The pure R-isomer ${ }^{13}$ of 1,2- $O$-ethylidene- $\beta$-D-mannopyranose was used for the synthesis of $\mathbf{2}$, giving predominantly the R-isomer of $\mathbf{2}$, and subsequently $\mathbf{3}$ as well.

2,3,4,6-Tetra-O-benzoyl- $\alpha$-D-mannopyranosyl-( $1 \rightarrow 3)$ 4,6-O-benzylidene-1,2-O-ethylidene- $\beta$-D-mannopyranose (3). - To a cooled solution $\left(0^{\circ} \mathrm{C}\right)$ of $1(7.40 \mathrm{~g}, 10$ $\mathrm{mmol}$ ) and 2 (R-form, $2.94 \mathrm{~g}, 10 \mathrm{mmol}$ ) in anhyd $\mathrm{CH}_{2} \mathrm{Cl}_{2}(50 \mathrm{~mL})$ was added TMSOTf $(50 \mu \mathrm{L}, 0.28$ $\mathrm{mmol}$ ). The mixture was stirred at this temperature for $2 \mathrm{~h}$, and then quenched with $\mathrm{Et}_{3} \mathrm{~N}$ ( 2 drops). The solvents were evaporated in vacuo to give a residue, which was purified by silica gel column chromatography (2:1 petroleum ether-EtOAc) to give disaccharide 3 as white foam $(4.72 \mathrm{~g}, 83 \%)$ : For R-isomer: $[\alpha]_{\mathrm{D}}$ $-80.6^{\circ}$ (c 1.0, $\mathrm{CHCl}_{3}$ ); ${ }^{1} \mathrm{H} \mathrm{NMR}\left(400 \mathrm{MHz}, \mathrm{CDCl}_{3}\right): \delta$ 8.07-7.37 (m, $25 \mathrm{H}, \mathrm{Ph} H), 6.07\left(\mathrm{dd}, 1 \mathrm{H}, J_{3,4}=J_{4,5} 9.8\right.$ Hz, H-4'), $6.01\left(\mathrm{dd}, 1 \mathrm{H}, J_{2,3} 3.0, J_{3,4} 9.8 \mathrm{~Hz}, \mathrm{H}-3^{\prime}\right), 5.86$ $\left(\mathrm{dd}, 1 \mathrm{H}, J_{1,2} 1.0, J_{2,3} 3.0 \mathrm{~Hz}, \mathrm{H}-2^{\prime}\right), 5.66(\mathrm{~s}, 1 \mathrm{H}$, $\mathrm{PhCH}), 5.59$ (d, $\left.1 \mathrm{H}, J_{1,2} 1.0 \mathrm{~Hz}, \mathrm{H}-1^{\prime}\right), 5.41(\mathrm{q}, 1 \mathrm{H}, J$ $4.7 \mathrm{~Hz}, \mathrm{MeCH}), 5.07$ (d, $\left.1 \mathrm{H}, J_{1,2} 1.6 \mathrm{~Hz}, \mathrm{H}-1\right), 4.69$ $\left(\mathrm{dd}, 1 \mathrm{H}, J_{2,3} 2.0, J_{3,4} 9.7 \mathrm{~Hz}, \mathrm{H}-3\right), 4.62$ (ddd, $1 \mathrm{H}, J_{4,5}$ 9.7, $\left.J_{5,6^{\prime} \mathrm{a}} 4.2, J_{5,6^{\prime} \mathrm{b}} 2.6 \mathrm{~Hz}, \mathrm{H}-5^{\prime}\right), 4.70\left(\mathrm{dd}, 1 \mathrm{H}, J_{5,6^{\prime}} 4.5\right.$, $\left.J_{6,6^{\prime}} 13.5 \mathrm{~Hz}, \mathrm{H}-6^{\prime}\right), 4.34-4.23\left(\mathrm{~m}, 4 \mathrm{H}, \mathrm{H}-2, \mathrm{H}-6^{\prime}\right.$ and 2 H-6), $3.81\left(\mathrm{dd}, 1 \mathrm{H}, J_{3,4}=J_{4,5} 9.7 \mathrm{~Hz}, \mathrm{H}-4\right), 3.36$ (ddd, $\left.1 \mathrm{H}, J_{4,5} 9.7, J_{5,6 \mathrm{a}} 4.5, J_{5,6 \mathrm{~b}} 4.3 \mathrm{~Hz}, \mathrm{H}-5\right), 1.58(\mathrm{~d}, 3 \mathrm{H}$, $J 4.7 \mathrm{~Hz}, M e \mathrm{CH})$. Anal. Calcd for $\mathrm{C}_{49} \mathrm{H}_{44} \mathrm{O}_{15}: \mathrm{C} 67.42$; H 5.08. Found: C 67.21; H 5.06.

2,3,4,6-Tetra-O-benzoyl- $\alpha$-D-mannopyranosyl-(1 $\rightarrow 3)$ 2,4,6-tri-O-acethyl- $\alpha$-D-mannopyranosyl trichloroacetimidate (7). - To a solution of $\mathbf{3}(7.58 \mathrm{~g}, 10.0 \mathrm{mmol})$ in anhyd $\mathrm{MeOH}(250 \mathrm{~mL})$ and $\mathrm{CH}_{2} \mathrm{Cl}_{2}(50 \mathrm{~mL})$ was added $\mathrm{AcCl}(1.5 \mathrm{~mL})$. The flask was stoppered, and the solution was stirred at $\mathrm{rt}$ for $1 \mathrm{~h}$, at the end of which time TLC (1:2 petroleum ether-EtOAc) showed that the starting material had disappeared. The solution was neutralized with $\mathrm{Et}_{3} \mathrm{~N}$, then concentrated to dryness. The residue was washed with water and extracted 3-4 times with $\mathrm{CH}_{2} \mathrm{Cl}_{2}$. The organic phase was dried over anhyd $\mathrm{Na}_{2} \mathrm{SO}_{4}$, then concentrated to dryness. The residue was passed through a short silica gel column (1:1.5 petroleum ether-EtOAc) to give $4(6.86 \mathrm{~g}, 90 \%)$ as a white solid. The white solid was dissolved in pyridine $(40 \mathrm{~mL})$, and then $\mathrm{Ac}_{2} \mathrm{O}(20 \mathrm{~mL})$ was added. After stirring the mixture at $\mathrm{rt}$ for $12 \mathrm{~h}$, TLC $(3: 2$ petroleum ether-EtOAc) indicated that the reaction was complete. The reaction mixture was concentrated to dryness. The resultant crude product 5 was dissolved in a $\mathrm{M}$ solution of $\mathrm{NH}_{3}-\mathrm{MeOH}(400 \mathrm{~mL})$ and stirred at $\mathrm{rt}$ until TLC (3:1 petroleum ether-EtOAc) indicated that the reaction was complete. The solution was concentrated to give compound $\mathbf{6}$ as a syrup. A mixture of 6, trichloroacetonitrile $(3.2 \mathrm{~mL}, 15 \mathrm{mmol})$, and 1,8-di- azabicyclo[5.4.0]undecene (DBU) $(0.50 \mathrm{~mL}, 4.04 \mathrm{mmol})$ in dry $\mathrm{CH}_{2} \mathrm{Cl}_{2}(50 \mathrm{~mL})$ was stirred under nitrogen for 3 $\mathrm{h}$ and then concentrated. The residue was purified by flash chromatography (2:1 petroleum ether-EtOAc) to give $7(5.55 \mathrm{~g}, 61 \%$ for three steps from 5 to 7$)$ as a white foam: $[\alpha]_{\mathrm{D}}-8.5^{\circ}\left(c 1.0, \mathrm{CHCl}_{3}\right) ;{ }^{1} \mathrm{H}$ NMR (400 $\left.\mathrm{MHz}, \mathrm{CDCl}_{3}\right): \delta 8.79(\mathrm{~s}, 1 \mathrm{H}, \mathrm{NH}), 8.15-7.21(\mathrm{~m}, 20$ $\mathrm{H}, 4 \mathrm{BzH}), 6.35\left(\mathrm{~d}, 1 \mathrm{H}, J_{1,2} 1.7 \mathrm{~Hz}, \mathrm{H}-1\right), 6.20(\mathrm{dd}, 1$ $\left.\mathrm{H}, J_{3,4}=J_{4,5} 9.9 \mathrm{~Hz}, \mathrm{H}-4^{\prime}\right), 5.78\left(\mathrm{dd}, 1 \mathrm{H}, J_{2,3} 3.2, J_{3,4}\right.$ $\left.9.9 \mathrm{~Hz}, \mathrm{H}-3^{\prime}\right), 5.59\left(\mathrm{dd}, 1 \mathrm{H}, J_{1,2} 1.4, J_{2,3} 3.2 \mathrm{~Hz}, \mathrm{H}-2^{\prime}\right)$, $5.54\left(\mathrm{dd}, 1 \mathrm{H}, J_{3,4}=J_{4,5} 10.0 \mathrm{~Hz}, \mathrm{H}-4\right), 5.48(\mathrm{dd}, 1 \mathrm{H}$, $\left.J_{1,2} 1.7, J_{2,3} 3.2 \mathrm{~Hz}, \mathrm{H}-2\right), 5.37\left(\mathrm{~d}, 1 \mathrm{H}, J_{1,2} 1.4 \mathrm{~Hz}\right.$, H-1'), 4.64-4.56 (m, $\left.2 \mathrm{H}, 2 \mathrm{H}-6^{\prime}\right), 4.47$ (dd, $1 \mathrm{H}, J_{5,6}$ 2.6, $\left.J_{6,6} 12.1 \mathrm{~Hz}, \mathrm{H}-6\right), 4.42\left(\mathrm{dd}, 1 \mathrm{H}, J_{2,3} 3.2, J_{3,4} 10.0\right.$ $\mathrm{Hz}, \mathrm{H}-3), 4.27$ (dd, $1 \mathrm{H}, J_{5,6} 5.2, J_{6,6} 12.4 \mathrm{~Hz}, \mathrm{H}-6$ ), 4.21-4.10 (m, 2 H, H-5, H-5'), 2.39 (s, 3 H, MeCO), 2.24 (s, $3 \mathrm{H}, M e \mathrm{CO}$ ), 2.10 (s, $3 \mathrm{H}, \mathrm{MeCO}$ ). Anal. Calcd for $\mathrm{C}_{48} \mathrm{H}_{44} \mathrm{Cl}_{3} \mathrm{NO}_{18}$ : C, 56.01; $\mathrm{H}, 4.31$. Found: $\mathrm{C}, 55.88$; $\mathrm{H}, 4.35$.

Allyl 3,4,6-tri-O-benzoyl- $\alpha$-D-mannopyranosyl- $(1 \rightarrow$ 2)-3,4,6-tri-O-benzoyl- $\alpha$-D-mannopyranoside (10).- - To a solution of $9(8.67 \mathrm{~g}, 10.0 \mathrm{mmol})$ in anhyd $\mathrm{MeOH}$ $(100 \mathrm{~mL})$ and $\mathrm{CH}_{2} \mathrm{Cl}_{2}(50 \mathrm{~mL})$ was added $\mathrm{AcCl}(0.5$ $\mathrm{mL}$ ). The flask was stoppered, and the solution was stirred at rt until TLC (3:1 petroleum ether-EtOAc) showed that the starting material disappeared (about $0.5-1 \mathrm{~h}$ ). The solution was neutralized with $\mathrm{Et}_{3} \mathrm{~N}$, then concentrated to dryness. The residue was passed through a short silica gel column (3:1 petroleum etherEtOAc) to give $\mathbf{1 0}(6.69 \mathrm{~g}, 80 \%)$ as a white solid: $[\alpha]_{\mathrm{D}}$ $-14.2^{\circ}\left(c 1.0, \mathrm{CHCl}_{3}\right) ;{ }^{1} \mathrm{H}$ NMR $\left(400 \mathrm{MHz}, \mathrm{CDCl}_{3}\right): \delta$ 8.14-7.24 (m, $30 \mathrm{H}, 6 \mathrm{BzH}), 5.97\left(\mathrm{dd}, 1 \mathrm{H}, J_{3,4}=J_{4,5}\right.$ $\left.9.9 \mathrm{~Hz}, \mathrm{H}-4^{\prime}\right), 5.90\left(\mathrm{dd}, 1 \mathrm{H}, J_{3,4}=J_{4,5} 10.0 \mathrm{~Hz}, \mathrm{H}-4\right)$, $5.88\left(\mathrm{~m}, 1 \mathrm{H}, \mathrm{CH}=\mathrm{CH}_{2}\right), 5.82\left(\mathrm{dd}, 1 \mathrm{H}, J_{2,3} 3.2, J_{3,4} 9.9\right.$ $\left.\mathrm{Hz}, \mathrm{H}-3^{\prime}\right), 5.79$ (dd, $1 \mathrm{H}, J_{2,3} 3.1, J_{3,4} 10.0 \mathrm{~Hz}, \mathrm{H}-3$ ), $5.26\left(\mathrm{dd}, 1 \mathrm{H}, J 1.5, J 17.2 \mathrm{~Hz}, \mathrm{CH}=\mathrm{CH}_{2}\right), 5.18(\mathrm{dd}, 1$ $\left.\mathrm{H}, J 1.5, J 10.6 \mathrm{~Hz}, \mathrm{CH}=\mathrm{CH}_{2}\right), 5.17\left(\mathrm{~d}, 1 \mathrm{H}, J_{1,2} 1.4 \mathrm{~Hz}\right.$, $\left.\mathrm{H}-1^{\prime}\right), 5.14\left(\mathrm{~d}, 1 \mathrm{H}, J_{1,2} 1.6 \mathrm{~Hz}, \mathrm{H}-1\right), 4.63-4.35$ (m, 8 H, H-2', H-2, H-5', H-5, 2 H-6', 2 H-6), 4.18 (dd, 1 H, $J$ 5.4, $\left.J 12.7 \mathrm{~Hz}, \mathrm{CH}_{2}-\mathrm{CH}=\mathrm{CH}_{2}\right), 3.92(\mathrm{dd}, 1 \mathrm{H}, J$ 6.0, $J 12.7 \mathrm{~Hz}, \mathrm{CH}_{2}-\mathrm{CH}=\mathrm{CH}_{2}$ ). Anal. Calcd for $\mathrm{C}_{57} \mathrm{H}_{50} \mathrm{O}_{17}$ : C, 67.99; H, 5.00. Found: C, 68.12; H, 5.03.

2,3,4,6-Tetra-O-benzoyl- $\alpha$-D-mannopyranosyl- $(1 \rightarrow 3)$ 2,4,6-tri-O-acetyl- $\alpha$-D-mannopyranosyl- $(1 \rightarrow 2)-3,4,6$ tri-O-benzoyl- $\alpha$-D-mannopyranosyl- $(1 \rightarrow 2)-3,4,6$-tri-Obenzoyl- $\alpha$-D-mannopyranosyl trichloroacetimidate (13). -Compound 11 (3.25 g, 61\%) was prepared by coupling of 7 (2.91 g, $2.83 \mathrm{mmol})$ with $\mathbf{1 0}(2.84 \mathrm{~g}, 2.82$ mmol) under the same conditions as described for the synthesis of $\mathbf{3}$ by coupling of $\mathbf{1}$ with $\mathbf{2}$. To a solution of $11(0.8 \mathrm{~g}, 0.43 \mathrm{mmol})$ in anhyd $\mathrm{MeOH}(20 \mathrm{~mL})$ was added $\mathrm{PdCl}_{2}(50 \mathrm{mg})$. After stirring the mixture at $\mathrm{rt}$ for $2 \mathrm{~h}$, TLC (3:2 petroleum ether-EtOAc) indicated that the reaction was complete. The mixture was filtered, and the solution was concentrated to dryness. The resultant residue was purified by flash chromatography 
(2:1 petroleum ether-EtOAc) to give $\mathbf{1 3}(0.6 \mathrm{~g}, 70 \%$ for two steps) as a white foam: $[\alpha]_{\mathrm{D}}-19.6^{\circ}\left(c 1.0, \mathrm{CHCl}_{3}\right)$; ${ }^{1} \mathrm{H}$ NMR $\left(400 \mathrm{MHz}, \mathrm{CDCl}_{3}\right): \delta 8.69(\mathrm{~s}, 1 \mathrm{H}, \mathrm{NH})$, 8.15-7.21 (m, $50 \mathrm{H}, 10 \mathrm{BzH}), 6.59\left(\mathrm{~d}, 1 \mathrm{H}, J_{1,2} 2.3 \mathrm{~Hz}\right.$, $\mathrm{H}-1), 6.24$ (dd, $\left.1 \mathrm{H}, J_{3,4}=J_{4,5} 10.1 \mathrm{~Hz}, \mathrm{H}-4^{\prime \prime \prime}\right), 6.05$ (dd, $\left.1 \mathrm{H}, J_{3,4}=J_{4,5} 10.3 \mathrm{~Hz}, \mathrm{H}-4^{\prime}\right), 5.97\left(\mathrm{dd}, 1 \mathrm{H}, J_{3,4}=J_{4,5}\right.$ $\left.10.1 \mathrm{~Hz}, \mathrm{H}-4^{\prime \prime}\right), 5.81\left(\mathrm{dd}, 1 \mathrm{H}, J_{2,3} 3.2, J_{3,4} 10.1 \mathrm{~Hz}\right.$, H-3"'), 5.78 (dd, $\left.1 \mathrm{H}, J_{2,3} 3.1, J_{3,4} 10.3 \mathrm{~Hz}, \mathrm{H}-3^{\prime}\right), 5.73$ $\left(\mathrm{dd}, 1 \mathrm{H}, J_{2,3} 3.2, J_{3,4} 10.1 \mathrm{~Hz}, \mathrm{H}-3^{\prime \prime}\right), 5.47-5.39$ (m, 3 $\left.\mathrm{H}, \mathrm{H}-1^{\prime \prime \prime}, \mathrm{H}-1^{\prime \prime}, \mathrm{H}-3\right), 5.30$ (d, $\left.1 \mathrm{H}, J_{1,2} 1.6 \mathrm{~Hz}, \mathrm{H}-1^{\prime}\right)$, $5.23\left(\mathrm{dd}, 1 \mathrm{H}, J_{3,4}=J_{4,5} 12.0 \mathrm{~Hz}, \mathrm{H}-4\right), 4.71-4.34(\mathrm{~m}$, 13 H, H-2"', H-2", H-2', H-2, H-5"', 2 H-6"', 2 H-6", 2 H-6', 2 H-6), 3.96 (m, 3 H, H-5", H-5', H-5), 2.22 (s, 3 $\mathrm{H}, \mathrm{MeCO}), 2.04$ (s, $3 \mathrm{H}, M e \mathrm{CO}), 2.02$ (s, $3 \mathrm{H}, M e \mathrm{CO}$ ). Anal. Calcd for $\mathrm{C}_{102} \mathrm{H}_{88} \mathrm{Cl}_{3} \mathrm{NO}_{34}$ : C, 61.93; $\mathrm{H}, 4.48$. Found: C, 61.78; H, 4.43.

Allyl 2,3,4-tri-O-benzoyl- $\alpha$-D-mannopyranosyl- $(1 \rightarrow$ 2) - 3,4,6- tri-O-benzoyl- $\alpha$-D - mannopyranosyl- $(1 \rightarrow 2)$ 3,4,6-tri-O-benzoyl- $\alpha$-D-mannopyranoside (16). - To a cooled solution $\left(0{ }^{\circ} \mathrm{C}\right)$ of $\mathbf{1 0}(1.01 \mathrm{~g}, 1 \mathrm{mmol})$ and $\mathbf{1 4}$ $(0.81 \mathrm{~g}, 1.2 \mathrm{mmol})$ in anhyd $\mathrm{CH}_{2} \mathrm{Cl}_{2}(50 \mathrm{~mL})$ was added TMSOTf $(30 \mu \mathrm{L}, 0.12 \mathrm{mmol})$. The mixture was stirred at $0{ }^{\circ} \mathrm{C}$ for $2 \mathrm{~h}$ and then quenched with $\mathrm{Et}_{3} \mathrm{~N}$ ( 2 drops). The solvent was evaporated to give a residue, which was purified by silica gel column chromatography $(2: 1$ petroleum ether-EtOAc) to give trisaccharide $\mathbf{1 5}$ as a foamy solid (1.24 g, 82\%). Compound $\mathbf{1 5}$ was dissolved in anhyd $\mathrm{MeOH}(200 \mathrm{~mL})$ and $\mathrm{CH}_{2} \mathrm{Cl}_{2}(100 \mathrm{~mL})$, and to the mixture was added $\mathrm{AcCl}(1.2 \mathrm{~mL})$. The flask was stoppered, and the solution was stirred at rt until TLC (1:1 petroleum ether-EtOAc) showed that the starting material disappeared $(2 \mathrm{~h})$. The solution was neutralized with $\mathrm{Et}_{3} \mathrm{~N}$, then concentrated to dryness. The residue was passed through a short silica gel column (1:2 petroleum ether-EtOAc) to give $16(810 \mathrm{mg}, 67 \%$ for two steps) as a white solid: $[\alpha]_{\mathrm{D}}-71.5^{\circ}(c$ 1.0, $\mathrm{CHCl}_{3}$ ); ${ }^{1} \mathrm{H}$ NMR (400 MHz, $\left.\mathrm{CDCl}_{3}\right): \delta 8.14-7.24(\mathrm{~m}$, $45 \mathrm{H}, 9 \mathrm{BzH}), 6.04-5.86$ (m, $5 \mathrm{H}, \mathrm{H}-3^{\prime \prime}, \mathrm{H}-4, \mathrm{H}-4^{\prime}$, $\mathrm{H}-4^{\prime \prime}, \mathrm{C} H=\mathrm{CH}_{2}$ ), 5.71-5.65 (m, $\left.3 \mathrm{H}, \mathrm{H}-3, \mathrm{H}-3^{\prime}, \mathrm{H}-2^{\prime \prime}\right)$, $5.39\left(\mathrm{~d}, 1 \mathrm{H}, J_{1,2} 1.5 \mathrm{~Hz}, \mathrm{H}-1^{\prime \prime}\right), 5.27(\mathrm{dd}, 1 \mathrm{H}, J 1.4, J$ $\left.17.2 \mathrm{~Hz}, \mathrm{CH}=\mathrm{CH}_{2}\right), 5.18(\mathrm{dd}, 1 \mathrm{H}, J 1.4, J 10.3 \mathrm{~Hz}$, $\left.\mathrm{CH}=\mathrm{CH}_{2}\right), 5.14\left(\mathrm{~d}, 1 \mathrm{H}, J_{1,2} 1.9 \mathrm{~Hz}, \mathrm{H}-1^{\prime}\right), 4.73(\mathrm{~d}, 1 \mathrm{H}$, $\left.J_{1,2} 1.0 \mathrm{~Hz}, \mathrm{H}-1\right), 4.63-4.50$ (m, $6 \mathrm{H}, \mathrm{H}-5^{\prime \prime}, 2 \mathrm{H}-6^{\prime \prime}, 2$ H-6', H-6),. 4.41-4.36 (m, 2 H, H-5', H-6), 4.18 (dd, 1 $\left.\mathrm{H}, J 6.0, J 12.7 \mathrm{~Hz}, \mathrm{CH}_{2}-\mathrm{CH}=\mathrm{CH}_{2}\right), 4.11$ (ddd, $1 \mathrm{H}, J_{4,5}$ $\left.11.3, J_{5,6 \mathrm{a}} 8.8, J_{5,6 \mathrm{~b}} 4.4 \mathrm{~Hz}, \mathrm{H}-5\right), 3.96(\mathrm{dd}, 1 \mathrm{H}, J 6.0, J$ $\left.12.7 \mathrm{~Hz}, \mathrm{CH}_{2}-\mathrm{CH}=\mathrm{CH}_{2}\right), 3.52\left(\mathrm{~m}, 2 \mathrm{H}, \mathrm{H}-2^{\prime}, \mathrm{H}-2\right) .{ }^{13} \mathrm{C}$ NMR $\left(100 \mathrm{MHz}, \mathrm{DCCl}_{3}\right): \delta 166.2,166.2,166.0,166.0$, $165.4,165.2,165.1,164.9,164.7$ (9 C, 9 PhCO), 133.5132.6, 130.0-128.2 (PhCO), $118.0 \quad(1 \quad \mathrm{C}$, $\left.-\mathrm{CH}_{2}-\mathrm{CH}=\mathrm{CH}_{2}\right), 100.4,99.7,98.0(3 \mathrm{C}, 3 \mathrm{C}-1), 71.9$, 71.9, 70.3, 70.0, 69.6, 69.3, 68.7, 68.7, 68.6, 67.3, 67.0, 66.7, 63.5, 63.5, 61.6, $61.4\left(\mathrm{C}-2-\mathrm{C}-6,-\mathrm{CH}_{2}-\mathrm{CH}=\mathrm{CH}_{2}\right)$. Anal. Calcd for $\mathrm{C}_{84} \mathrm{H}_{72} \mathrm{O}_{25}: \mathrm{C}, 68.10 ; \mathrm{H}, 4.90$. Found: C, 67.90; H, 4.87.
Allyl 2,3,4,6-tetra-O-benzoyl- $\alpha$-D-mannopyranosyl$(1 \rightarrow 3)$-2,4,6-tri-O-acethyl- $\alpha$-D-mannopyranosyl-(1 $\rightarrow 2)$ 3,4,6-tri-O-benzoyl- $\alpha$-D-mannopyranosyl-(1 $\rightarrow 2)$-3,4,6tri-O-benzoyl- $\alpha$-D-mannopyranosyl- $(1 \rightarrow 6)-2,3,4-$ tri-Obenzoyl- $\alpha$-D-mannopyranosyl- $(1 \rightarrow 2)-3,4,6$ - tri-O-benzoyl- $\alpha$-D-mannopyranosyl- $(1 \rightarrow 2)$-3,4,6-tri-O-benzoyl$\alpha$-D-mannopyranoside (17). - Under the same conditions as described for the synthesis of $\mathbf{3}$ by coupling of 1 with 2, heptasaccharide 17 (94 mg, 51\%) was obtained from coupling of $\mathbf{1 3}(150 \mathrm{mg}, 0.076 \mathrm{mmol})$ with $\mathbf{1 6}$ (100 $\mathrm{mg}, 0.067 \mathrm{mmol}):[\alpha]_{\mathrm{D}}-19.6^{\circ}\left(c \quad 1.0, \mathrm{CHCl}_{3}\right) ;{ }^{1} \mathrm{H}$ NMR $\left(400 \mathrm{MHz}, \mathrm{CDCl}_{3}\right): \delta 8.19-7.20(\mathrm{~m}, 95 \mathrm{H}, 9$ BzH), 6.31-6.18 (m, 2 H, 2 H-4), 6.08-5.63 (m, $11 \mathrm{H}$, $\left.\mathrm{H}-2,4 \mathrm{H}-3,5 \mathrm{H}-4, \mathrm{CH}=\mathrm{CH}_{2}\right), 5.51-5.15(\mathrm{~m}, 10 \mathrm{H}, 4$ $\left.\mathrm{H}-1, \mathrm{H}-2,3 \mathrm{H}-3,2 \mathrm{CH}=\mathrm{CH}_{2}\right), 4.81-4.33(\mathrm{~m}, 22 \mathrm{H}, 3$ H-1, 3 H-2, 4 H-5, 12 H-6), 4.30-4.08 (m, 5 H, 2 H-5, $\left.2 \mathrm{H}-6, \mathrm{CH}_{2}-\mathrm{CH}=\mathrm{CH}_{2}\right), 4.06-3.68$ (m, $4 \mathrm{H}, 2 \mathrm{H}-2, \mathrm{H}-5$, $\mathrm{CH}_{2}-\mathrm{CH}=\mathrm{CH}_{2}$ ), 2.19 (s, $\left.3 \mathrm{H}, \mathrm{MeCO}\right), 1.94$ (s, $3 \mathrm{H}$, $M e \mathrm{CO}), 1.85$ (s, $3 \mathrm{H}, M e \mathrm{CO}) .{ }^{13} \mathrm{C}$ NMR $(100 \mathrm{MHz}$, $\left.\mathrm{CDCl}_{3}\right): \delta 170.1,169.8,169.6(3 \mathrm{C}, 3 \mathrm{MeCO}), 166.2$, $165.9,165.8,165.7,165.6,165.5,165.3,165.2,165.1$, 165.0, 164.9 (19 C, $\mathrm{PhCO}$ ), 133.7-132.2, 130.2-129.2, 129.1-127.9 $\left(\mathrm{PhCO},-\mathrm{CH}_{2}-\mathrm{CH}=\mathrm{CH}_{2}\right), 118.0$ (1 C, $\left.-\mathrm{CH}_{2}-\mathrm{CH}=\mathrm{CH}_{2}\right), 100.6$, 99.8, 99.8, 99.7, 99.0, 98.3, 98.0 (7 C-1), 77.3 (C-3'"'"'), 73.4, 72.2, 71.3, 70.8, 70.6, $70.3,70.1,70.0,69.7,69.6,69.4,69.2,68.7,68.5,67.2$, $66.3,65.9,65.8,63.6,63.5,62.9,62.8,62.2,62.0$ (C-2C-6, $\left.-\mathrm{CH}_{2}-\mathrm{CH}=\mathrm{CH}_{2}\right), 21.4,20.7,20.5$ (3 Me CO). Anal. Calcd for $\mathrm{C}_{184} \mathrm{H}_{158} \mathrm{O}_{58}$ : C, 67.03; H, 4.83. Found: C, $67.10 ; \mathrm{H}, 4.82$.

Allyl $\alpha$-D-mannopyranosyl-(1 $\rightarrow 3)-\alpha$-D-mannopyranosyl- $(1 \rightarrow 2)-\alpha$-D-mannopyranosyl- $(1 \rightarrow 2)-\alpha$-D-mannopyranosyl - $(1 \rightarrow 6)-\alpha-\mathrm{D}$ - mannopyranosyl - $(1 \rightarrow 2)-\alpha-\mathrm{D}-$ mannopyranosyl-( $1 \rightarrow 2)-\alpha$-D-mannopyranoside (18).Compound 17 (75 mg, $0.0275 \mathrm{mmol}$ ) was dissolved in satd $\mathrm{NH}_{3}-\mathrm{MeOH}(10 \mathrm{~mL})$. After 2 weeks at rt, the reaction solution was concentrated, and the residue was purified on a BioGel P2 column with $\mathrm{MeOH}$-water as the eluent to afford $\mathbf{1 8}(24 \mathrm{mg}, 80 \%)$ as a pulverous crystalloid: $[\alpha]_{\mathrm{D}}+52.6^{\circ}$ (c 1.0, water); ${ }^{1} \mathrm{H}$ NMR (400 $\left.\mathrm{MHz}, \mathrm{D}_{2} \mathrm{O}\right): \delta 5.87\left(\mathrm{~m}, 1 \mathrm{H}, \mathrm{CH}=\mathrm{CH}_{2}\right), 5.25(\mathrm{dd}, 1 \mathrm{H}$, $\left.J 1.6, J 17.2 \mathrm{~Hz}, \mathrm{CH}=\mathrm{CH}_{2}\right), 5.20,5.18,5.10,5.05,5.04$ $(5 \mathrm{H}, \mathrm{H}-1), 5.01\left(\mathrm{dd}, 1 \mathrm{H}, J 1.6, J 10.8 \mathrm{~Hz}, \mathrm{CH}=\mathrm{CH}_{2}\right)$, 4.94, 4.94 (2 H, H-1), 4.18-3.40 (m, $44 \mathrm{H}$, $\left.\mathrm{CH}_{2}-\mathrm{CH}=\mathrm{CH}_{2}, \mathrm{H}-2-\mathrm{H}-6\right) .{ }^{13} \mathrm{C}$ NMR $\left(100 \mathrm{MHz}, \mathrm{D}_{2} \mathrm{O}\right)$ : $\delta \quad 132.7 \quad\left(1 \quad \mathrm{C}, \quad-\mathrm{CH}_{2}-\mathrm{CH}=\mathrm{CH}_{2}\right), \quad 117.9 \quad(1 \quad \mathrm{C}$, $\left.-\mathrm{CH}_{2}-\mathrm{CH}=\mathrm{CH}_{2}\right), 101.7,101.7,101.6,100.3,100.0,97.7$, $96.9\left(7 \mathrm{C}-1, J_{\mathrm{C}-1, \mathrm{H}-1} 172.0-173.5 \mathrm{~Hz}\right), 78.2,78.1,78.0$, 77.8, 77.4, 75.4, 75.2, 75.0, 73.6, 72.9, 72.8, 72.8, 72.4, $72.3,72.2,71.5,71.1,70.2,70.0,70.0,69.8,69.7,69.5$, 69.4, 69.1, 67.6, 66.5, 66.4, 66.3, 65.8, 65.6, 61.9, 60.5, $60.4,60.2,60.2 \quad\left(\mathrm{C}-2-\mathrm{C}-6,-\mathrm{CH}_{2}-\mathrm{CH}=\mathrm{CH}_{2}\right)$. Anal. Calcd for $\mathrm{C}_{45} \mathrm{H}_{76} \mathrm{O}_{36}: \mathrm{C}, 45.30 ; \mathrm{H}, 6.42$. Found: $\mathrm{C}$, 45.17; H, 6.47. 


\section{Acknowledgements}

This work was supported by The Chinese Academy of Sciences (Projects KJ952J $\mathrm{J}_{1} 510$ and KIPRCEES9904) and by The National Natural Science Foundation of China (Projects 39970864 and 30070815).

\section{References}

1. Goodman, D. S.; Teplitz, E. D.; Wishner, A.; Klein, R. S.; Burk, P. G.; Hershenbaum, E. J. Am. Acad. Dermatol. 1987, 17, 210-218.

2. Bodey, G. P. Am. J. Med. 1984, 77, 13-22.

3. Spebar, M. J.; Pruitt, B. A. J. Trauma 1981, 21, 237-243.

4. Silverman, S., Jr.; Luangjarmekorn, L.; Greenspan, D. J. Oral Med. 1984, 39, 194-201.

5. Goldman, W. E. Science 1999, 285, 539-541.

6. Kobayashi, H.; Mitobe, H.; Takahashi, K.; Yamamoto,
T.; Shibata, N.; Suzuki, S. Arch. Biochem. Biophys. 1992, 294, 662-669.

7. Schmidt, R. R.; Kinzy, W. Adv. Carbohydr. Chem. Biochem. 1994, 50, 21-125.

8. (a) Zhu, Y.; Kong, F. Synlett 2000, 1783-1788;

(b) Zhu, Y.; Kong, F. Chin. J. Chem. 2001, 19, 119-123.

9. (a) Auzanneau, F.-I.; Forooghian, F.; Pinto, B. M. Carbohydr. Res. 1996, 291, 21-40;

(b) Wang, W.; Kong, F. Carbohydr. Res. 1999, 315, $128-135$.

10. Ogawa, T.; Yamamoto, H. Carbohydr. Res. 1985, 137, $79-87$.

11. Heng, L.; Ning, J.; Kong, F. J. Carbohydr. Chem. 2001, 20, 285-296.

12. (a) Nakahara, Y.; Shibayama, S.; Nakahara, Y.; Ogawa, T. Carbohydr. Res. 1996, 280, 67-84;

(b) Grice, P.; Ley, S. V.; Pietrunzka, J. Angew. Chem., Int. Ed. Engl. 1996, 35, 197-200;

(c) Ogawa, T.; Sasajima, K. Tetrahedron 1981, 37, $2787-$ 2792.

13. Betaneli, V. I.; Ovchinnikov, M. V.; Kochetkov, N. K. Carbohydr. Res. 1982, 107, 285-291. 